\title{
Hypoxia in the microenvironment promotes glycolysis to aggravate tumor progression via modulating the lincRNA- p21 and its downstream genes in HCC
}

\author{
Type
}

Research paper

\section{Keywords}

Hypoxia, hepatocellular carcinoma, glycolysis, miR-181b, HK2, lincRNA-p21

\begin{abstract}
Introduction

LincRNA-p21 was found to inhibit hepatic stellate cell (HSC) activation and liver fibrosis via a signaling cascade of lincRNA-p21-miR-181b-PTEN. Hypoxia was also previously proved to regulate hepatocellular carcinoma (HCC) glycolysis by targeting HK2.

Material and methods

Luciferase assay was carried out to examine the regulatory role of miR-181b in lincRNA-p21 and HK2 expression. Quantitative real-time PCR was performed to measure the expression of lincRNA$\mathrm{p} 21, \mathrm{miR}-181 \mathrm{~b}$ and HK2 mRNA. Western blot and immunohistochemistry were used to analyze the expression of HK2 protein.
\end{abstract}

\section{Results}

The expression of lincRNA-p21 and HK2 was effectively suppressed by miR-181b in Hep3B and HepG2 cells. Besides, the luciferase activities of wild type lincRNA-p21 and HK2 were remarkably suppressed by miR-181b in Hep3B and HepG2 cells. Activation and suppression of lincRNA-p21 expression using pcDNA and shRNA revealed a negative correlation between miR-181b and lincRNA-p21 expression as well as a positive correlation between HK2 and lincRNA-p21 expression. Moreover, lincRNA-p21 shRNA could effectively reverse the effect of hypoxia-induced dysregulation in miR-181b and HK2 expression, as well as the altered levels of glucose consumption and lactate production in Hep3B and HepG2 cells. Furthermore, lincRNA-p21 was capable of altering the growth and miR-181b/HK2 expression of HepG2 xenograft tumors in nude mice.

\section{Conclusions}

Our study investigated the molecular relationship between lincRNA-p21, miR-181b and HK2 in cellular and animal models, and validated that hypoxia could up-regulate the expression level of lincRNA-p21 in the microenvironment of solid hepatocellular carcinoma tumor, which accordingly led to aggravated glycolysis via elevated HK2 expression, thus inhibiting the apoptosis of HCC. 
Hypoxia in the microenvironment promotes glycolysis to aggravate tumor progression via modulating the lincRNA-p21 and its downstream genes in HCC

Shu-jie Li ${ }^{1}$, Ding-yi Yang ${ }^{1}$, Fu-rong Wu ${ }^{1}$, Wei Zhou ${ }^{2}$, Yue Xie ${ }^{1 *}$, Ying Wang ${ }^{2 *}$

1. Chongqing Key Laboratory of Translational Research for Cancer Metastasis and Individualized Treatment, Department of Radiation Oncology, Chongqing University Cancer Hospital \& Chongqing Cancer Institute \& Chongqing Cancer Hospital, Chongqing, 400030, China

2. Chongqing Key Laboratory of Translational Research for Cancer Metastasis and Individualized Treatment, Chongqing University Cancer Hospital \& Chongqing Cancer Institute \& Chongqing Cancer Hospital, Chongqing, 400030, China

Corresponding authors:

Ying Wang, Chongqing Key Laboratory of Translational Research for Cancer Metastasis and Individualized Treatment, Chongqing University Cancer Hospital \& Chongqing Cancer Institute \& Chongqing Cancer Hospital, No. 181 Hanyu Road, Shapingba District, Chongqing, 400030, China,surgdocx@yeah.net

Yue Xie, Chongqing Key Laboratory of Translational Research for Cancer Metastasis and Individualized Treatment, Department of Radiation Oncology, Chongqing University Cancer Hospital \& Chongqing Cancer Institute \& Chongqing Cancer Hospital, No. 181 Hanyu Road, Shapingba District, Chongqing, 400030, China,

Email: surgdocx@yeah.net

\section{Abstract}

Background: LincRNA-p21 was found to inhibit hepatic stellate cell (HSC) activation and liver fibrosis via a signaling cascade of lincRNA-p21-miR-181b-PTEN. Hypoxia was also previously proved to regulate hepatocellular carcinoma (HCC) glycolysis by targeting HK2. Methods: Luciferase assay was carried out to examine the regulatory role of miR-181b in lincRNA-p21 and HK2 expression. Quantitative real-time PCR was performed to measure the expression of lincRNA-p21, miR-181b and HK2 mRNA. Western blot and immunohistochemistry were used to analyze the expression of HK2 protein. Results: The expression of lincRNA-p21 and HK2 was 
effectively suppressed by miR-181b in Hep3B and HepG2 cells. Besides, the luciferase activities of wild type lincRNA-p21 and HK2 were remarkably suppressed by miR-181b in Hep3B and HepG2 cells. Activation and suppression of lincRNA-p21 expression using pcDNA and shRNA revealed a negative correlation between miR-181b and lincRNA-p21 expression as well as a positive correlation between HK2 and lincRNA-p21 expression. Moreover, lincRNA-p21 shRNA could effectively reverse the effect of hypoxia-induced dysregulation in miR-181b and HK2 expression, as well as the altered levels of glucose consumption and lactate production in Hep3B and HepG2 cells. Furthermore, lincRNA-p21 was capable of altering the growth and miR181b/HK2 expression of HepG2 xenograft tumors in nude mice. Conclusion: Our study investigated the molecular relationship between lincRNA-p21, miR-181b and HK2 in cellular and animal models, and validated that hypoxia could up-regulate the expression level of lincRNA-p21 in the microenvironment of solid hepatocellular carcinoma tumor, which accordingly led to aggravated glycolysis via elevated HK2 expression, thus inhibiting the apoptosis of HCC.

Running title: Hypoxia aggravates tumor progression

Keywords: Hypoxia, hepatocellular carcinoma, lincRNA-p21, miR-181b, HK2, glycolysis

\section{Abbreviation}

HCC: hepatocellular carcinoma

HK2: hexokinase 2

\section{Introduction}

Since 2008, hepatocellular carcinoma has ranked one of the top 10 most common malignancies in the adult population [1]. Almost 1 million new cases of HCCs are detected every year, with almost $80 \%$ of the cases are caused by hepatitis B and hepatitis C viral infections [2]. For example, in some developing countries, hepatitis $\mathrm{B}$ and hepatitis $\mathrm{C}$ infections are responsible for a higher ratio of HCC cases than those in industrialized nations [3]. Thus, high HCC incidence has been a challenge in sub-Saharan Africa, Southeast Asia, and East Asia, especially in China [4].

Oxygen is crucial for maintaining a normal metabolic rate in mammals [5,6]. Since the key reactions in the mitochondria mainly include energy generation by sugar or fat reaction via converting adenosine 5-triphosphate from adenosine 5-diphosphate, therefore, as a state of low 
oxygen supply, hypoxia adjusts the metabolic demands of cells. Depending on the ability of cells to adapt to hypoxia, the cells can tolerate hypoxia or commit apoptosis $[7,8]$.

Numerous long non-coding RNAs (lncRNAs), such as lincRNA-p21 and H19, have recently been demonstrated to be moderated by hypoxia and involved in the signaling transduction of cancer cells $[9,10]$. In a recent research, it was revealed that in a hypoxic environment, lincRNA-p21 expression is induced by hypoxia-inducible factor-1 alpha (HIF-1a) and in turn stabilizes the expression level of HIF-1a, thereby creating positive feedback to maintain HIF-1a expression [11].

LncRNA molecules usually contain more than 200 nt but do not possess a protein-coding functionality [12]. LncRNAs are involved in the control of cell growth and migration. LncRNAs can also control the transcription of genes through modulation of chromatin, the regulation at the post-transcriptional level, the formation of protein complex, and protein regulation at the allosteric level [13].

In a previous research, it was presented that lincRNA-p21 blocked the activation of HSC by mediating the expression of miR-181b and phosphatase and tensin homolog (PTEN) [14].

As a key isozyme expressed richly in a number of different types of cancers cells, Hexokinase 2 (HK2) also promotes aerobic glycolysis by inducing the Warburg effect. Thus, HK2 has been studied as a target for the treatment of cancers $[15,16]$. It was also discovered that miR-181b reduces glycolysis through the suppression of protein expression of HK2. Consequently, miR$181 \mathrm{~b}$ was determined as a key regulator in the metabolism of glucose in cancer cells [17].

Nevertheless, from the result of a GO enrichment analysis on genes associated with glycolysis, it was shown that the only HCC showed a strong correlation with the level of glycolysis since the golgi apparatus was responsible for the effect of glycolysis on HCC [18].

The level of glycolysis may be increased to a certain extent through the re-expression of WT HK2, but it can never fully return to the normal level through the use of either GCK or a mutant with a mitochondrial binding deficiency (MTD), illustrating that the binding of HK2 to mitochondria is required to exert the glycolytic effect of HK2 in cells [19].

In a previous report on lincRNA-p21, hypoxic preconditioning was demonstrated to be a factor promoting the migration and survival of mesenchymal stem cells (MSC) via affecting lincRNA- 
p21 expression [20]. And lincRNA-p21 was found to inhibit hepatic stellate cell (HSC) activation and liver fibrosis via a signaling cascade of lincRNA-p21-miR-181b-PTEN [14]. Also, hypoxia was previously proved to regulate HCC glycolysis by targeting HK2 [21]. In this study, we studied the effect of hypoxia on tumorigenesis of HCC by modulating glycolysis via regulating the expression of lincRNA-p21 using animal xenograft model as well as in vitro analysis. With the in vitro assay, we studied the regulatory relationship between lincRNA-p21, miR-181b and HK2. Also, the cells were subjected to hypoxia treatment to study the effect of hypoxia upon the lincRNA-p21 axis as well as the role of lincRNA-p21 during hypoxia in an ex vivo model. Moreover, we also established animal xenograft model to validate the effect of hypoxia and underlying molecular mechanism in HCC tumorigenesis in an in vivo model.

\section{Materials and Methods}

\section{Cell culture and transfection}

In order to examine the effect of lincRNA-p21 on hypoxia, Hep3B and HepG2 cells were subjected to hypoxia treatment followed by lincRNA-p21 shRNA transfection. Hep3B and HepG2 cells were cultured in the DMEM medium (Gibco, Thermo Fisher Scientific, Waltham, MA) added with 10\% FBS and suitable antibiotics. To induce the hypoxic culture conditions, the cells were placed in an MIC-101 incubator (Billups-Rothenberug, Del Mar, CA) pre-purged using 5\% CO2 and 95\% N2. The cells were cultured under the above hypoxic culture conditions for $48 \mathrm{~h}$ at $37^{\circ} \mathrm{C}$. The media used in both hypoxic and normal culture conditions were the same. In this study, several cellular models were established. In cellular model 1, Hep3B and HepG2 cells were divided into 2 groups, i.e., 1. NC group (Hep3B and HepG2 cells transfected with $\mathrm{NC}$ vector); and 2. miR-181b precursor group (Hep3B and $\mathrm{HepG} 2$ cells transfected with the vector carrying the miR-181b precursor). In cellular model 2, Hep3B and HepG2 cells were also divided into 2 groups, i.e., 1. pcDNA group (Hep3B and HepG2 cells transfected with an empty pcDNA vector); and 2. pcDNA-lincRNA-p21 group (Hep3B and HepG2 cells transfected with the pcDNA vector carrying lincRNA-p21). In cellular model 3, Hep3B and HepG2 cells were also divided into 2 groups, i.e., 1. NC shRNA group (Hep3B and HepG2 cells transfected with NC shRNA); and 2. LincRNA-p21 shRNA group (Hep3B and HepG2 cells transfected with LincRNA-p21 shRNA). In cellular model 4, Hep3B and HepG2 cells were divided into 3 groups, i.e., 1. Normoxia group (Hep3B and HepG2 cells cultured under normal culture conditions); 2. Hypoxia+ NC shRNA group (Hep3B and HepG2 cells 
115

116

117

118

119

120

121

122

123

124

125

126

127

128

129

130

131

132

133

134

135

136

137

138

139

140

141

142

143

144

cultured under hypoxic culture conditions and transfected with NC shRNA); and 3. Hypoxiat LincRNA-p21 shRNA group (Hep3B and HepG2 cells cultured under hypoxic culture conditions and transfected with LincRNA-p21 shRNA). All transfections were carried out using Lipofectamine 2000 (Invitrogen, Carlsbad, CA) based on the instructions provided by the transfection reagent manufacturer, and all transfected cells were harvested $48 \mathrm{~h}$ post-transfection to assay target genes.

\section{Vector construction mutagenesis and luciferase assay}

Our binding sites screening of miR-181b showed that miR-181b could potentially target lincRNAp21 and the 3' UTR of HK2. In order to further validate the regulatory relationship between miR$181 \mathrm{~b}$ and its target genes, luciferase assays were carried out. In brief, wild type sequences of lincRNA-p21 and 3' UTR of HK2 containing the miR-181b binding sites were cloned into pcDNA-6.2 plasmid vectors (Invitrogen, Carlsbad, CA) based on the instructions provided by the plasmid vector manufacturer, so as to create the wild type plasmids for lincRNA-p21 and 3' UTR of HK2, respectively. On the other hand, site-directed mutagenesis was carried out at the miR$181 \mathrm{~b}$ binding sites using a Quick Change mutagenesis assay kit (Stratagene, San Diego, CA) based on the instructions provided by the assay kit manufacturer, and the mutant type sequences of lincRNA-p21 and 3' UTR of HK2 were also cloned into pcDNA-6.2 plasmid vectors to create the mutant type plasmids for lincRNA-p21 and 3' UTR of HK2, respectively. In the next step, luciferase vectors containing wild type and mutant lincRNA-p21 and HK2 were co-transfected into Hep3B and HepG2 cells along with miR-181b. The transfections were carried out using Lipofectamine 2000 (Invitrogen, Carlsbad, CA) based on the instructions provided by the transfection reagent manufacturer, and the luciferase activity of transfected cells was measured on a luminometer using the Bright-Glo luciferase assay kit (Promega, Madison, WI) $48 \mathrm{~h}$ later based on the instructions provided by the transfection reagent manufacturer.

\section{RNA isolation and real-time PCR}

Total RNA enriched of miRNA and lincRNA was separated from the cell and tissue samples by making use of a QIAzol assay kit (Qiagen, Valencia, CA) in conjunction with a miRNeasy assay kit (Qiagen, Valencia, CA) based on the instructions provided by the assay kit manufacturer. The ratio of absorbance at 260/280 was used to measure total RNA concentration on a SmartSpec 300 Spectrophotometer (Bio-Rad Laboratories, Hercules, CA) based on the instructions provided by 
the instrument manufacturer. Then, the cDNA synthesis was conducted by using a First Strand assay kit (Qiagen, Valencia, CA) based on the instructions provided by the assay kit manufacturer. In the next step, the synthesized cDNA was used as the template to perform real-time PCR by using an SYBR Green qPCR Master Mix (Qiagen, Valencia, CA) based on the instructions provided by the assay kit manufacturer. The real-time PCR reactions were carried out in 96-well MAH-001A miFinder PCR assay plate (Qiagen, Valencia, CA) on a MyiQ Cycler (Bio-Rad Laboratories, Hercules, CA) based on the instructions provided by the instrument manufacturer. Finally, the expression of lincRNA-p21, miR-181b, and HK2 mRNA in each sample was calculated by using the threshold cycle $(\mathrm{Ct})$ number of their amplification curves, and the calculated expression of lincRNA-p21, miR-181b, and HK2 mRNA was normalized to that of the housekeeping gene GAPDH.

\section{Western blot analysis}

Total protein was isolated from tissue and cell samples by lysis in a RIPA buffer (Sigma-Aldrich, St. Louis, MO) based on the instructions provided by the buffer manufacturer. Then, the protein samples were subject to $30 \mathrm{~min}$ of centrifugation at $4^{\circ} \mathrm{C}$ and $600 \times \mathrm{g}$ to eliminate cell debris. The concentrations of isolated protein samples were measured by using a BCA assay kit (Bio-Rad Laboratories, Hercules, CA) based on the instructions provided by the assay kit manufacturer, and an equal amount of isolated protein from each sample was resolved by 10\% SDS-PAGE and blotted onto a PVDF membrane (Millipore, Bedford, MA), which was then blocked by using 5\% skim milk and probed with primary and suitable horseradish peroxidase-conjugated secondary antibodies against HK2 based on the incubation instructions provided by the antibody manufacturer (Abcam, Cambridge, MA). After PBST washing, the PVDF membrane was developed by using enhanced chemiluminescence (ECL) assay kit (GE Healthcare, Buckinghamshire, England) based on the instructions provided by the assay kit manufacturer. The relative HK2 protein expression in each sample was then determined densitometrically by using a Kodak imager (Kodak Molecular Image Resolution, Rochester, NY) based on the instructions provided by the instrument manufacturer.

\section{Animal and treatment}

In order to examine the effect of lincRNA-p21 on tumor, HepG2 cells were transfected with lincRNA-p21 shRNA and transplanted into nude mice. In this study, a total of 15 male BALB/c 
175 athymic nude mice of 3-4 weeks in age were acquired from Beijing HFK Bioscience (Beijing, 176 China) and then housed under pathogen-free conditions. All animal procedures were done based 177 on the Guide for the Care and Use of Laboratory Animals published by the NIH. After 7 days of 178 environmental adaptation, the $\mathrm{BALB} / \mathrm{c}$ athymic nude mice were divided into 2 groups with 179 randomly selecting 7 mice into each group, i.e., 1. NC shRNA group (BALB/c athymic nude mice transplanted with HepG2 cells transfected with NC shRNA), and 2. LincRNA-p21 shRNA group 181 (BALB/c athymic nude mice transplanted with HepG2 cells transfected with LincRNA-p21 182 shRNA). During the transplantation procedure, the HepG2 cells were subcutaneously injected into the left side of mice, with $4 \times 10^{6}$ cells injected into each mouse. Eight days after the transplantation procedure, it was shown that the tumors in the mice had different sizes. The tumor volume in each mouse was calculated as length $\times$ width $^{2} / 2$. At the end of the experiment, tumor tissues from all mice were harvested for immunohistochemical assays. The institutional ethical committee has approved the protocol of this study.

\section{Immunohistochemistry}

189 Collected tissue samples were fixed in paraformaldehyde, embedded in paraffin, sliced into 5 um 190 sections, deparaffinized, dehydrated with gradient alcohol, and then incubated with primary antiHK2 antibody (1:200; Abcam, Cambridge, MA) and biotin-conjugated secondary antibodies in 192 conjunction with a PV6001 PowerVision Two-Step Histostaining Reagent (ZSGB, Beijing, China) 193 based on the instructions provided by the assay kit manufacturer. After counterstaining with a hematoxylin and diaminobenzidine (DAB) assay kit (ZSGB, Beijing, China) based on the instructions provided by the assay kit manufacturer, the slides were visualized by utilizing a 196 microscope (Olympus, Tokyo, Japan).

\section{Measurement of glucose consumption and lactate production}

198 Glucose consumption and lactate production were measured following protocols provided by a 199 previous publication [22]. Supernatants of cell culture media were collected. And the glucose and 200 lactate levels were measured using a Glucose Assay kit (Sigma-Aldrich St. Louis, MO) and a 201 Lactate Assay kit (Sigma-Aldrich St. Louis, MO) according to the manufacturer's instructions.

\section{Statistical analysis}


224

225

226

227

228

229

230

All data were statistically evaluated using the Sigma Stat and Sigma Plot Software (Systat Software, Chicago, IL). Inter-group variations were evaluated by using a one-way analysis of variance (ANOVA). A p-value of $<0.05$ was considered statistically significant. All data were expressed as mean \pm standard error.

\section{Results}

MiR-181b effectively suppressed the expression of lincRNA-p21 and HK2 in Hep3B and HepG2 cells.

Binding sites screening of miR-181b showed that miR-181b could potentially target lincRNA-p21 and the 3' UTR of HK2. In order to further validate the regulatory relationship between miR-181b and its target genes, luciferase vectors containing wild type and mutant lincRNA-p21 and HK2 were established and transfected into Hep3B and HepG2 cells along with miR-181b. The luciferase activities of wild type lincRNA-21b were remarkably suppressed by miR-181b in Hep3B and HepG2 cells (Fig.1A). The luciferase activities of wild type HK2 were significantly inhibited by miR-181b in Hep3B and HepG2 cells (Fig.1B). No notable repression was observed for mutant lincRNA-p21 and HK2 in either Hep3B or HepG2 cells. Moreover, miR-181b precursors were transfected into Hep3B and HepG2 cells to examine their effect on the expression of lincRNA-p21 and HK2. A dramatic increase in miR-181b expression in Hep3B and HepG2 cells indicated successful transfection of miR-181b precursors (Fig.1C). The expression of lincRNA-p21 was significantly decreased by miR-181b precursors in Hep3B and HepG2 cells when compared with the control (Fig.1D). Similarly, the expression of HK2 mRNA (Fig.1E) and protein (Fig.1F) was apparently suppressed in Hep3B and HepG2 cells transfected with miR-181b precursors.

\section{LincRNA-p21 overexpression decreased miR-181b level and increased HK2 level as well as glucose consumption and lactate production in Hep3B and HepG2 cells}

Furthermore, we overexpressed lincRNA-p21 in Hep3B and HepG2 cells (Fig.2A) and checked the expression of miR-181b and $\mathrm{HK} 2 \mathrm{mRNA} /$ protein, as well as the levels of glucose consumption and lactate in the supernatant of cell culture. The expression of miR-181b was evidently suppressed in Hep3B and HepG2 cells by lincRNA-p21 (Fig.2B). However, the expression of HK2 mRNA (Fig.2C) and protein (Fig.2D) was significantly enhanced by lincRNA-p21 overexpression in Hep3B and HepG2 cells when compared with the control. Glucose assay and lactate assay showed 
that the overexpression of lincRNA-p21 remarkably increased the levels of glucose consumption (Fig.2E) and lactate production (Fig.2F) in the supernatant of cell culture.

\section{Suppression of lincRNA-p21 activated the expression of miR-181b and decreased the} expression of $\mathrm{HK} 2$ and the levels of glucose consumption and lactate in the supernatant of Hep3B and HepG2 cells.

Moreover, we suppressed the expression of lincRNA-p21 in Hep3B and HepG2 cells using lincRNA-p21 shRNA (Fig.3A). The expression of miR-181b and HK2, as well as the glucose consumption and lactate levels in the supernatant were further evaluated. LincRNA-p21 shRNA significantly enhanced the expression of miR-181b in Hep3B and HepG2 cells (Fig.3B), whereas the expression of HK2 mRNA (Fig.3C) and protein (Fig.3D) was notably suppressed by lincRNAp21 shRNA in Hep3B and HepG2 cells. Besides, the glucose consumption (Fig.3E) and lactate (Fig.3F) levels in the supernatant of cell culture were diminished by lincRNA-p21 shRNA in Hep3B and HepG2 cells.

LincRNA-p21 shRNA attenuated hypoxia-induced dysregulation of miR-181b and HK2 expression, as well as the levels of glucose consumption and lactate production in Hep3B and HepG2 cells.

In order to examine the effect of lincRNA-p21 on hypoxia, Hep3B and HepG2 cells were subjected to hypoxia treatment followed by lincRNA-p21 shRNA transfection. Hypoxia notably activated the expression of lincRNA-p21 in Hep3B and HepG2 cells, while lincRNA-p21 shRNA attenuated hypoxia-induced up-regulation of lincRNA-p21 expression (Fig.4A). Hypoxia remarkably suppressed the expression of miR-181b, while lincRNA-p21 shRNA restored the expression of miR-181b to a certain level in Hep3B and HepG2 cells (Fig.4B). Besides, the levels of HK2 expression (Fig.4C, D), as well as glucose consumption (Fig.4E) and lactate production (Fig.4F) elevated by hypoxia were effectively decreased by lincRNA-p21 shRNA in Hep3B and HepG2 cells.

LincRNA-p21 shRNA altered the volume of HepG2 tumor tissue and weight of nude mice, as well as the expression of miR-181b and HK2 in nude mice.

HepG2 cells were transfected with lincRNA-p21 shRNA and transplanted into nude mice. The solid tissue was harvested and subjected to size evaluation. And the tumor tissue volume (Fig.5A) 
were significantly reduced in the group transplanted with HepG2 cells transfected with lincRNAp21 shRNA, while no difference of body weight was observed between the mice groups (Fig.5B). The expression of lincRNA-p21 in the HepG2 tumor tissue treated with lincRNA-p21 shRNA was significantly decreased when compared with the control (Fig.5C), whereas the expression of miR181b was increased (Fig.5D). Quantitative real-time PCR showed that the expression of HK2 mRNA was suppressed in the HepG2 tumor tissue treated with lincRNA-p21 shRNA (Fig.5E). Western blot and immunohistochemistry analysis indicated that the expression of HK2 protein was effectively inhibited in the HepG2 tumor tissue treated with lincRNA-p21 shRNA (Fig.5F, Fig.6).

\section{Discussion}

In this study, we transfected HepG2 cells with lincRNA-p21 shRNA and then transplanted the cells into nude mice, and checked the tumor volume and the weight of the mice, as well as the expression of lincRNA-p21, miR-181b, and HK2 in tumor tissues. LincRNA-p21 shRNA remarkably decreased the tumor volume and the weight of the nude mice transplanted with HepG2 cells. Increased expression of miR-181b and suppressed expression of HK2 was observed in HepG2 tumor tissues treated with lincRNA-p21 shRNA.

Hypoxia can trigger cell adaptations at the translational, transcriptional, post-translational, and epigenetic levels to reduce the generation of ROS and the expenditure of ATP, so as to maintain the homeostasis and survival ability of cells [23]. Mechanistic studies have disclosed that HK-2 plays an essential role in chrysin to show its effect in the HCC. Along with the decreased level of HK-2 after treatment with chrysin, the level of glycolysis in the HCC was also noticeably inhibited. In samples of human HCC, the protein expression of HIF1 $\alpha$ was substantially raised to cause a poorer prognosis [24-26]. In addition, the expression of HIF $1 \alpha$ in HCC tumors has been used as a factor to predict patient survival [26, 27]. In this study, we performed binding sites screening of miR-181b and used luciferase assays to explore the suppressive role of miR-181b in lincRNA-p21 and HK2 expression in Hep3B and HpeG2 cells. MiR-181b effectively inhibited the expression of lincRNA-p21 and HK2 in Hep3B and HepG2 cells.

While some previous studies have presented that lincRNA-p21 can respond to hypoxia to act as a cell cycle regulator by inducing apoptosis and the Warburg effect in certain cancer cells, the biological role of lincRNA-p21 in hepatoma and glioma under hypoxic conditions remains unclear. In one study, it was found that the treatment with hypoxia raised the expression of lincRNA-p21 
in U251MG glioma and SMMC7721 hepatoma cells [28]. In addition, lincRNA-p21 was actually determined to inhibit the metastasis and invasion of HCC cells via the epithelial-mesenchymal transition (EMT) mechanism [29]. In this study, we altered the expression of lincRNA-p21 using lincRNA-p21 pcDNA and shRNA. The expression of lincRNA-p21 was positively correlated with the expression of HK2 and negatively correlated with the expression of miR-181b in Hep3B and HepG2 cells.

It was presented that miR-181b was most noticeably downregulated in human NSCLC cells [30]. Significantly, reduced miR-181b expression enhances the proliferation and chemoresistance of human NSCLC cells against cisplatin [31, 32]. Another research stated that miR-181b was involved in the chemo resistance of U87 glioma cells against temozolomide, suggesting a role of miR-181b in the control of chemo sensitivity [33].

MiR-181b was also shown to be downregulated in glioma to hinder the proliferation, invasion, and migration of many types of cancer cells by targeting the IGF-1 signaling [34, 35]. Additionally, miR-181b could improve the sensitivity of drugs in myeloid leukemia by inhibiting glycolysis and the Warburg effect [17, 36].

The HK2 enzyme plays a critical role in the glycolytic signaling of cancer by catalyzing the initial step of glycolysis [15, 37]. The HK2 expression in cancer cells was shown to be higher than that in normal cells, suggesting that HK2 may be used as a target for the development of cancer therapy [38-40]. In this study, we found that the down-regulation of lincRNA-p21 expression significantly attenuated hypoxia-induced dysregulation of miR-181b and HK2 expression in Hep3B and HepG2 cells.

Since it was shown that the systemic HK2 deletion in adult mice had no visible side effect 9, HK2 might act as an ideal target in the HCC treatment. It was also discovered that the ablation of HK2 in HCC cells inhibited their survival and proliferation. HK2 is a primary isoform in the skeletal muscular tissues, heart, and adipose tissues. HK2 is additionally upregulated in lots of tumors linked to aerobic glycolysis. [40]. One study suggested that GPC3 is considerably involved in glucose metabolism reprogramming via HIF-1 $\alpha$-induced expression of Glut1, HK2, and LDHA enzymes to downregulate the expression of PGC-1 $\alpha$, a regulator in the biogenesis of mitochondria [41]. 


\section{Conclusion}

321 In conclusion, our study established the molecular regulatory relationships between lincRNA-p21,

322 miR-181b and HK2 in cellular and animal models and validated that hypoxia could up-regulate

323 the expression of lincRNA-p21 in the microenvironment of solid hepatocellular carcinoma tumor,

324 which accordingly led to aggravated glycolysis via elevated HK2 expression, thus inhibiting the

325 apoptosis of HCC.

326 Conflict of interest

327 None

$328 \quad$ Funding statement

329 This study was supported by Chongqing Science and Technology Bureau (ID: cstc2018jxj10068)

330 and Chongqing Science and Technology Innovation Guidance Project Led by Academician (ID: 331 cstc2017jcyj-yszx0001).

332 Availability of data and material

333 The data that support the findings of this study are available from the corresponding author upon 334 reasonable request.

335 Author contributions

336 YW, YX and SJL planned the study and performed the majority of the laboratory work, DYY, 337 FRW and WZ collected and analyzed the data and participated in writing. All authors conceived 338 and designed the experiments and drafted the manuscript.

\section{$339 \quad$ Figure legends}

$340 \quad$ Fig. 1

341 MiR-181b effectively suppressed the expression of lincRNA-p21 and HK2 in Hep3B and HepG2 342 cells (* P-value < 0.05 compared with NC group; NC: negative control).

343 A: Sequence analysis and luciferase assay showed that the luciferase activities of wild type 344 lincRNA-p21 were suppressed by miR-181b in Hpe3B and HepG2 cells. 
B: Sequence analysis and luciferase assay showed that the luciferase activities of wild type HK2 were suppressed by miR-181b in Hpe3B and HepG2 cells.

C: The expression of miR-181b was remarkably elevated in Hep3B and HepG2 cells transfected with miR-181b precursors.

D: The expression of lincRNA-p21 was notably suppressed in Hep3B and HepG2 cells transfected with miR-181b precursors.

E: The expression of HK2 mRNA was remarkably suppressed in Hep3B and HepG2 cells transfected with miR-181b precursors.

F: The expression of HK2 protein was remarkably suppressed in Hep3B and HepG2 cells transfected with miR-181b precursors.

\section{Fig.2}

Overexpression of lincRNA-p21 decreased the expression of miR-181, enhanced the expression of HK2 mRNA and protein, and promoted glucose consumption and lactate production in Hep3B and HepG2 cells (* P-value < 0.05 compared with pcDNA group; pcDNA functions as the control group).

A: Expression of lincRNA-p21 was dramatically elevated in Hep3B and HepG2 cells transfected with pcDNA-lincRNA-p21.

B: The expression of miR-181b was remarkably suppressed by lincRNA-p21 overexpression in Hep3B and HepG2 cells.

C: The expression of HK2 mRNA was remarkably increased by lincRNA-p21 overexpression in Hep3B and HepG2 cells.

D: The expression of HK2 protein was remarkably increased by lincRNA-p2 overexpression 1 in Hep3B and HepG2 cells.

E: The glucose consumption was notably promoted by lincRNA-p21 overexpression in Hep3B and HepG2 cells.

F: The lactate production was notably promoted by lincRNA-p21 overexpression in Hep3B and HepG2 cells. 
$372 \quad$ Fig.3

373 Suppression of lincRNA-p21 enhanced the expression of miR-181, repressed the expression of 374 HK2 mRNA and protein, and reduced the levels of glucose consumption and lactate production (*

375 P-value < 0.05 compared with NC shRNA group; NC: negative control; NC shRNA functions as 376 the control group).

377 A: Dramatic decrease of lincRNA-p21 expression in Hep3B and HepG2 cells transfected with 378 lincRNA-p21 shRNA.

379 B: The expression of miR-181b was remarkably increased by lincRNA-p21 shRNA in Hep3B and 380 HepG2 cells.

381 C: The expression of HK2 mRNA was remarkably decreased by lincRNA-p21 shRNA in Hep3B 382 and HepG2 cells.

383 D: The expression of HK2 protein was remarkably decreased by lincRNA-p21 shRNA in Hep3B 384 and HepG2 cells.

385 E: The glucose consumption was notably suppressed by lincRNA-p21 shRNA in Hep3B and 386 HepG2 cells.

387 F: The lactate production was notably suppressed by lincRNA-p21 shRNA in Hep3B and HepG2 388 cells.

$389 \quad$ Fig.4

390 LincRNA-p21 shRNA attenuated hypoxia-induced dysregulation of miR-181b and HK2 391 expression, as well as the levels of glucose consumption and lactate production in Hep3B and 392 HepG2 cells (* P-value < 0.05 compared with Normoxia group; ** $\mathrm{P}$ value < 0.05 compared with 393 Hypoxia + NC shRNA group; NC: negative control).

394 A: Hypoxia induced up-regulation of lincRNA-p21 was decreased by lincRNA-p21 shRNA in 395 Hep3B and HepG2 cells.

396 B: Hypoxia induced down-regulation of miR-181b was decreased by lincRNA-p21 shRNA in 397 Hep3B and HepG2 cells. 
C: Hypoxia induced up-regulation of HK2 mRNA was decreased by lincRNA-p21 shRNA in 399 Hep3B and HepG2 cells.

400 D: Hypoxia induced up-regulation of HK2 protein was decreased by lincRNA-p21 shRNA in 401 Hep3B and HepG2 cells.

402 E: Hypoxia induced elevation of glucose consumption was decreased by lincRNA-p21 shRNA in 403 Hep3B and HepG2 cells.

404 F: Hypoxia induced elevation of lactate production was decreased by lincRNA-p21 shRNA in 405 Hep3B and HepG2 cells.

$406 \quad$ Fig.5

407 HepG2 cells treated by lincRNA-p21 shRNA altered the volume of tumor tissues and weight of 408 nude mice, as well as the expression of miR-181b and HK2 (* P-value < 0.05 compared with NC 409 shRNA group; NC: negative control; NC shRNA functions as the control group).

410 A: LincRNA-p21 shRNA remarkably decreased the tumor volume of the nude mice transplanted 411 with HepG2 cells.

412 B: LincRNA-p21 shRNA did not significantly influence the body weight of the nude mice 413 transplanted with HepG2 cells.

414 C: The expression of lincRNA-p21 was decreased in the HepG2 tumor tissue treated by lincRNA415 p21 shRNA.

416 D: The expression of miR-181b was increased in the HepG2 tumor tissue treated by lincRNA-p21 417 shRNA.

418 E: The expression of HK2 mRNA was decreased in the HepG2 tumor tissue treated by lincRNA419 p21 shRNA.

420 F: Western blot analysis showed that the expression of HK2 protein was decreased in the HepG2 421 tumor tissue treated by lincRNA-p21 shRNA.

$422 \quad$ Fig.6 
423 Immunohistochemistry analysis showed that the expression of HK2 protein was decreased in the

424 HepG2 tumor tissue treated by lincRNA-p21 shRNA (The blue stain denotes the nucleus and the 425 brown stain denotes the expression of target protein HK2).

\section{References}

4271 Jemal A, Bray F, Center MM, Ferlay J, Ward E, Forman D: Global cancer statistics. CA $428 \quad$ Cancer J Clin 2011;61:69-90.

4292 Bejarano Ramírez DF, Carrasquilla Gutiérrez G, Porras Ramírez A, Vera Torres A: $430 \quad$ Prevalence of liver disease in Colombia between 2009 and 2016. JGH Open 2020; $431 \quad 19 ; 4(4): 603-610$.

4323 Parkin DM: The global health burden of infection-associated cancers in the year 2002. Int $433 \quad$ J Cancer 2006;118:3030-3044.

4344 Richman DM, Tirumani SH, Hornick JL, Fuchs CS, Howard S, Krajewski K, Ramaiya N, 435 Rosenthal M: Beyond gastric adenocarcinoma: Multimodality assessment of common and 4375 Severinghaus JW, Astrup P, Murray JF: Blood gas analysis and critical care medicine. Am 438 J Respir Crit Care Med 1998;157:S114-122.

440 pulmonary circulation. J Thorac Cardiovasc Surg 1975;70:229-236.

4417 Leach RM, Treacher DF: Oxygen transport-2. Tissue hypoxia. BMJ 1998;317:1370-1373.

4428 Martin DS, Khosravi M, Grocott MP, Mythen MG: Concepts in hypoxia reborn. Crit Care $443 \quad 2010 ; 14: 315$.

4449 Matouk IJ, DeGroot N, Mezan S, Ayesh S, Abu-lail R, Hochberg A, Galun E: The H19 445 non-coding RNA is essential for human tumor growth. PLoS One 2007;2:e845.

44610 Yang F, Huo XS, Yuan SX, Zhang L, Zhou WP, Wang F, Sun SH: Repression of the long 447 noncoding RNA-LET by histone deacetylase 3 contributes to hypoxia-mediated metastasis. 448 44911 Yang F, Zhang H, Mei Y, Wu M: Reciprocal regulation of HIF-1alpha and lincRNA-p21 450 modulates the Warburg effect. Mol Cell 2014;53:88-100.

$451 \quad 12$ Wilusz JE, Sunwoo H, Spector DL: Long noncoding RNAs: functional surprises from the 452 RNA world. Genes Dev 2009;23:1494-1504. 
45313 Geisler S, Coller J: RNA in unexpected places: long non-coding RNA functions in diverse $454 \quad$ cellular contexts. Nat Rev Mol Cell Biol 2013;14:699-712.

45514 Yu F, Lu Z, Chen B, Dong P, Zheng J: Identification of a Novel lincRNA-p21-miR-181b456 PTEN Signaling Cascade in Liver Fibrosis. Mediators Inflamm 2016;2016:9856538.

45715 Mathupala SP, Ko YH, Pedersen PL: Hexokinase-2 bound to mitochondria: cancer's 458 stygian link to the "Warburg Effect" and a pivotal target for effective therapy. Semin $459 \quad$ Cancer Biol 2009;19:17-24.

46016 Vander Heiden MG: Targeting cancer metabolism: a therapeutic window opens. Nat Rev 461 Drug Discov 2011;10:671-684.

46217 Li LQ, Yang Y, Chen H, Zhang L, Pan D, Xie WJ: MicroRNA-181b inhibits glycolysis in 463 gastric cancer cells via targeting hexokinase 2 gene. Cancer Biomark 2016;17:75-81.

46418 Sin AT, Harrison RE: Growth of the Mammalian Golgi Apparatus during Interphase. Mol 465 Cell Biol 2016;36:2344-2359.

467

20 Meng SS, Xu XP, Chang W, Lu ZH, Huang LL, Xu JY, Liu L, Qiu HB, Yang Y, Guo FM: LincRNA-p21 promotes mesenchymal stem cell migration capacity and survival through hypoxic preconditioning. Stem Cell Res Ther 2018;9:280.

474

21 Jin F, Wang Y, Zhu Y, Li S, Liu Y, Chen C, Wang X, Zen K, Li L: The miR-125a/HK2 axis regulates cancer cell energy metabolism reprogramming in hepatocellular carcinoma. Sci Rep 2017;7:3089. regulates glycolysis via targeting hexokinase 2 in hepatocellular carcinoma cells. Oncotarget 2017;8(15):24777-24784. Biol 2018;28:128-142. of HIF-1alpha and HIF-2alpha in hepatocellular carcinoma with capsular invasion, portal vein tumor thrombi and patients' clinical outcome. Jpn J Clin Oncol 2014;44:159-167. 
25 Bangoura G, Liu ZS, Qian Q, Jiang CQ, Yang GF, Jing S: Prognostic significance of HIF2alpha/EPAS1 expression in hepatocellular carcinoma. World J Gastroenterol 2007;13:3176-3182.

26 Dai CX, Gao Q, Qiu SJ, Ju MJ, Cai MY, Xu YF, Zhou J, Zhang BH, Fan J: Hypoxiainducible factor-1 alpha, in association with inflammation, angiogenesis and MYC, is a critical prognostic factor in patients with HCC after surgery. BMC Cancer 2009;9:418.

27 Xiang ZL, Zeng ZC, Fan J, Tang ZY, He J, Zeng HY, Chang JY: The expression of HIF1alpha in primary hepatocellular carcinoma and its correlation with radiotherapy response and clinical outcome. Mol Biol Rep 2012;39:2021-2029.

28 Shen Y, Liu Y, Sun T, Yang W: LincRNA-p21 knockdown enhances radiosensitivity of hypoxic tumor cells by reducing autophagy through HIF-1/Akt/mTOR/P70S6K pathway. Exp Cell Res 2017;358:188-198.

29 Ding G, Peng Z, Shang J, Kang Y, Ning H, Mao C: LincRNA-p21 inhibits invasion and metastasis of hepatocellular carcinoma through miR-9/E-cadherin cascade signaling pathway molecular mechanism. Onco Targets Ther 2017;10:3241-3247.

30 Yang J, Liu H, Wang H, Sun Y: Down-regulation of microRNA-181b is a potential prognostic marker of non-small cell lung cancer. Pathol Res Pract 2013;209:490-494.

31 Wang X, Chen X, Meng Q, Jing H, Lu H, Yang Y, Cai L, Zhao Y: MiR-181b regulates cisplatin chemosensitivity and metastasis by targeting TGFbetaR1/Smad signaling pathway in NSCLC. Sci Rep 2015;5:17618.

32 Zhu W, Shan X, Wang T, Shu Y, Liu P: miR-181b modulates multidrug resistance by targeting BCL2 in human cancer cell lines. Int J Cancer 2010;127:2520-2529.

33 Li P, Lu X, Wang Y, Sun L, Qian C, Yan W, Liu N, You Y, Fu Z: MiR-181b suppresses proliferation of and reduces chemoresistance to temozolomide in U87 glioma stem cells. J Biomed Res 2010;24:436-443.

34 Shi ZM, Wang XF, Qian X, Tao T, Wang L, Chen QD, Wang XR, Cao L, Wang YY, Zhang JX, Jiang T, Kang CS, Jiang BH, Liu N, You YP: MiRNA-181b suppresses IGF$1 \mathrm{R}$ and functions as a tumor suppressor gene in gliomas. RNA 2013;19:552-560.

35 Li D, Jian W, Wei C, Song H, Gu Y, Luo Y, Fang L: Down-regulation of miR-181b promotes apoptosis by targeting CYLD in thyroid papillary cancer. Int J Clin Exp Pathol 2014;7:7672-7680. 
51536 Cai B, An Y, Lv N, Chen J, Tu M, Sun J, Wu P, Wei J, Jiang K, Miao Y: miRNA-181b

516 increases the sensitivity of pancreatic ductal adenocarcinoma cells to gemcitabine in vitro $517 \quad$ and in nude mice by targeting BCL-2. Oncol Rep 2013;29:1769-1776.

51837 Hanahan D, Weinberg RA: Hallmarks of cancer: the next generation. Cell 2011;144:646519674.

52038 Ros S, Schulze A: Glycolysis back in the limelight: systemic targeting of HK2 blocks $521 \quad$ tumor growth. Cancer Discov 2013;3:1105-1107.

52239 Patra KC, Wang Q, Bhaskar PT, Miller L, Wang Z, Wheaton W, Chandel N, Laakso M, 523 Muller WJ, Allen EL, Jha AK, Smolen GA, Clasquin MF, Robey B, Hay N: Hexokinase 2 524 is required for tumor initiation and maintenance and its systemic deletion is therapeutic in 525 mouse models of cancer. Cancer Cell 2013;24:213-228.

52640 Tan VP, Miyamoto S: HK2/hexokinase-II integrates glycolysis and autophagy to confer $527 \quad$ cellular protection. Autophagy 2015;11:963-964.

52841 Yao G, Yin J, Wang Q, Dong R, Lu J: Glypican-3 Enhances Reprogramming of Glucose 529 Metabolism in Liver Cancer Cells. Biomed Res Int 2019;2019:2560650. 
A

lincRNA-p21 WT 5' GCCTGCTG ACAGCCAGAGAG GGTA 3' miR-181b | | | | || || | | || || lincRNA-p21 MUT 5' GTT TG TGTGTGTT C CCATGGGGTA 3,

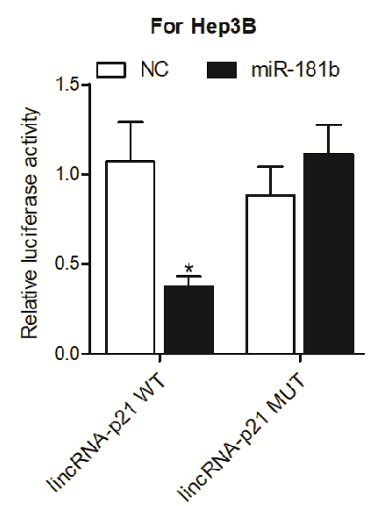

C

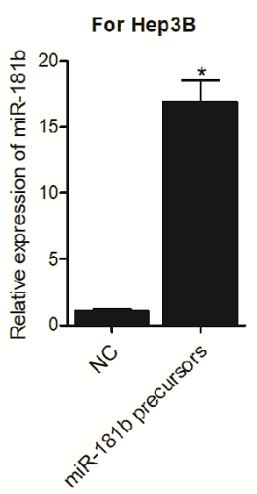

E

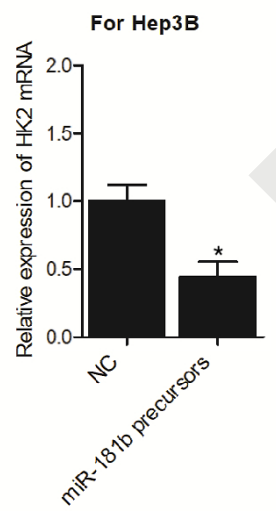

For HepG2
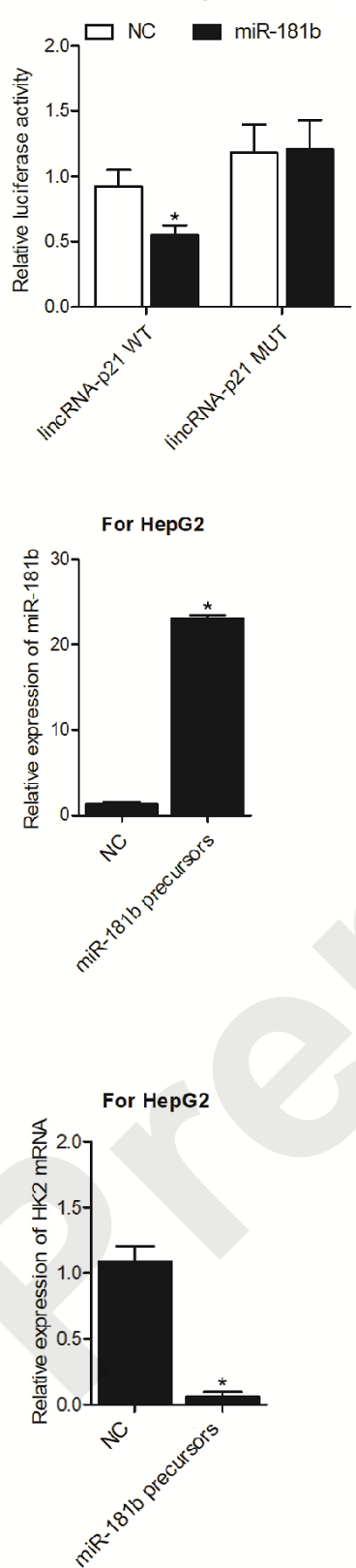

B

HK2 3'UTR WT 5' TTGTAAGT TT TAA ATAA AAGGTC 3

|| ||| ||

3. AACAUUCAUUGCUGUCGGUGGGU 5' I | || |

HK2 3'UTR MUT 5' TTCAAAC TTTT AAAT AA AAGGTC 3
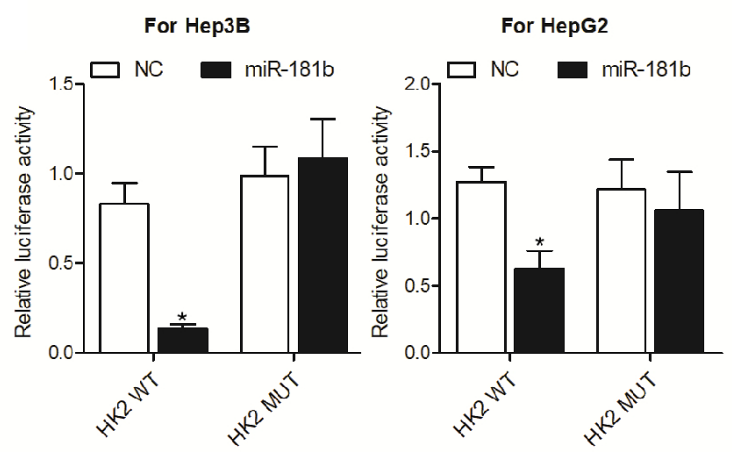

D
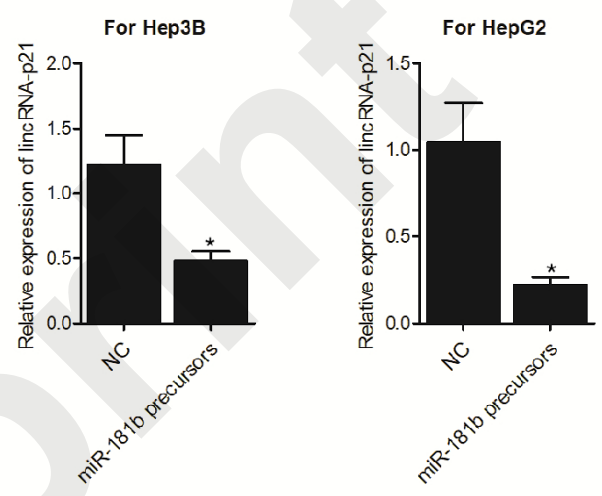

F
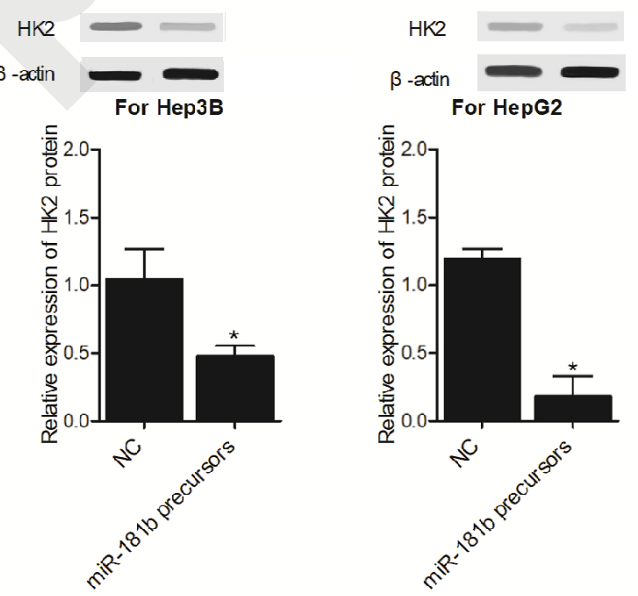

Fig. 1

MiR-181b effectively suppressed the expression of lincRNA-p21 and HK2 in Hep3B and HepG2 cells ( ${ }^{*}$ P-value < 0.05 compared with NC group; NC: negative control).

$A$ : Sequence analysis and luciferase assay showed that the luciferase activities of wild type lincRNA-p21 were suppressed by miR-181b in Hpe3B and HepG2 cells.

$B$ : Sequence analysis and luciferase assay showed that the luciferase activities of wild type HK2 were suppressed by miR-181b in Hpe3B and HepG2 cells.

C: The expression of miR-181b was remarkably elevated in Hep3B and HepG2 cells transfected with miR-181b precursors. 
D: The expression of lincRNA-p21 was notably suppressed in Hep3B and HepG2 cells transfected with miR-181b precursors.

E: The expression of HK2 mRNA was remarkably suppressed in Hep3B and HepG2 cells transfected with miR-181b precursors.

F: The expression of HK2 protein was remarkably suppressed in Hep3B and HepG2 cells transfected with miR-181b precursors. 
A

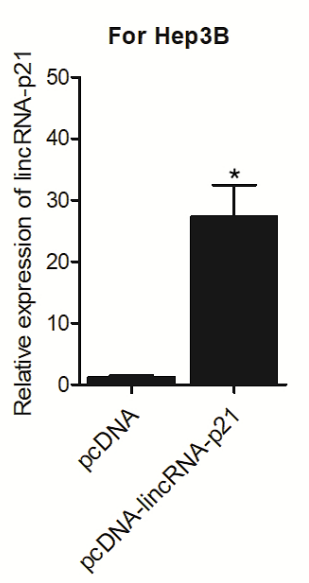

C

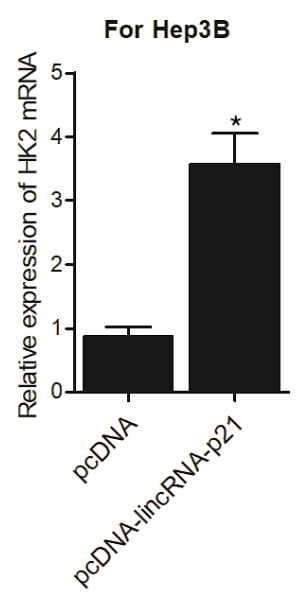

E

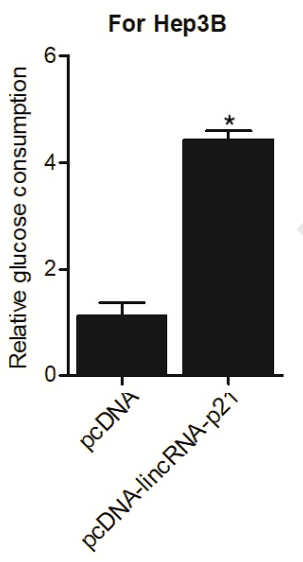

For HepG2

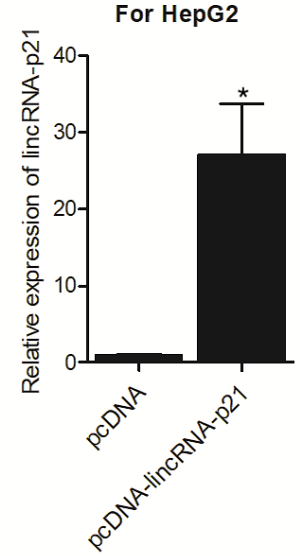

D

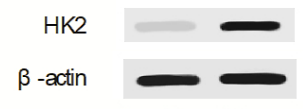

For Hep3B

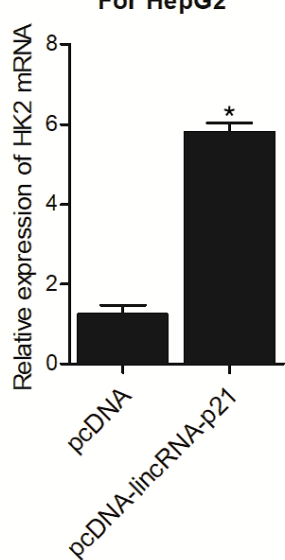

$\mathbf{F}$
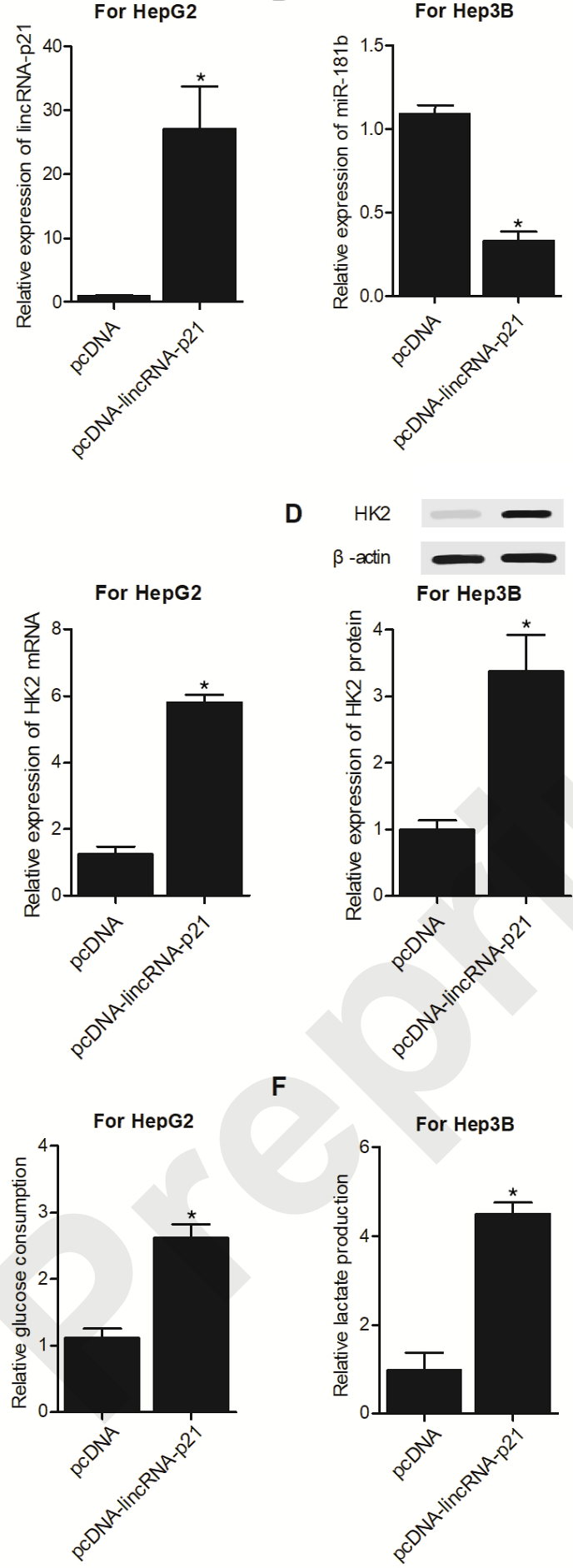

B
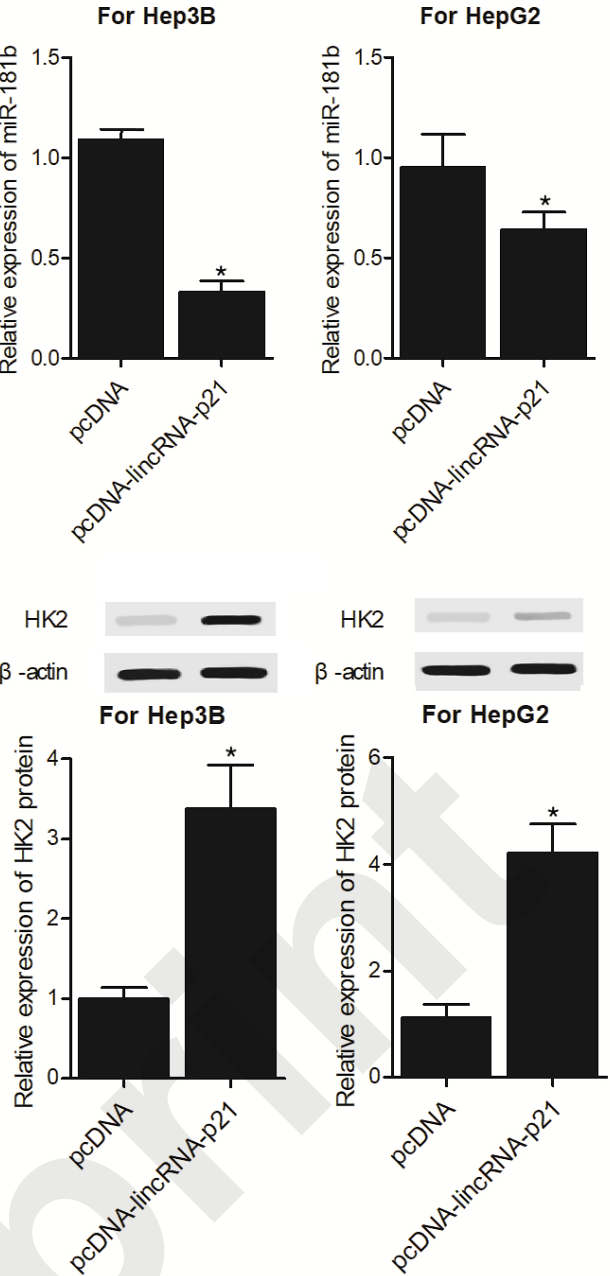

Fig.2

Overexpression of lincRNA-p21 decreased the expression of miR-181, enhanced the expression of HK2 mRNA and protein, and promoted glucose consumption and lactate production in Hep3B and HepG2 cells ( ${ }^{*} P$-value $<0.05$ compared with pcDNA group; pcDNA functions as the control group).

A: Expression of lincRNA-p21 was dramatically elevated in Hep3B and HepG2 cells transfected with pcDNA-lincRNA-p21.

$B$ : The expression of miR-181b was remarkably suppressed by lincRNA-p21 overexpression in Hep3B and HepG2 cells. 
C: The expression of HK2 mRNA was remarkably increased by lincRNA-p21

overexpression in Hep3B and HepG2 cells.

D: The expression of HK2 protein was remarkably increased by lincRNA-p2 overexpression 1 in Hep3B and HepG2 cells.

$\mathrm{E}$ : The glucose consumption was notably promoted by lincRNA-p21 overexpression in Hep3B and HepG2 cells.

$F$ : The lactate production was notably promoted by lincRNA-p21 overexpression in Hep3B and HepG2 cells. 
A

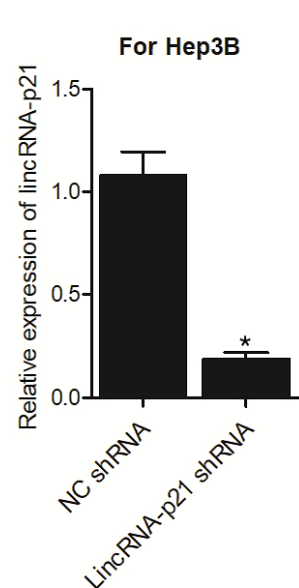

C

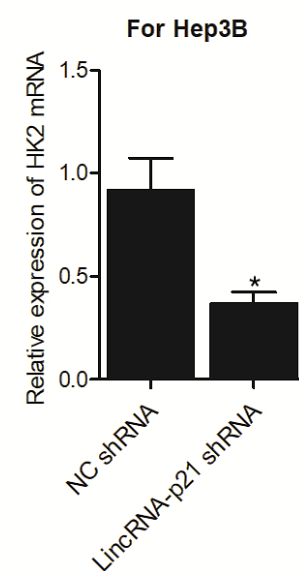

E

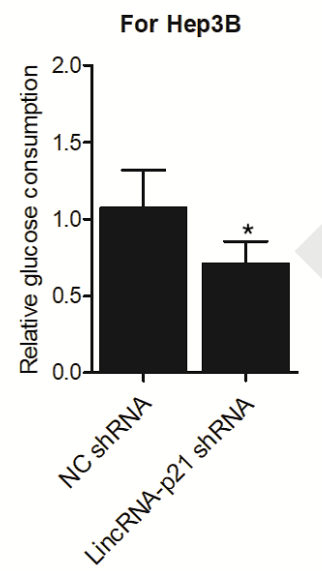

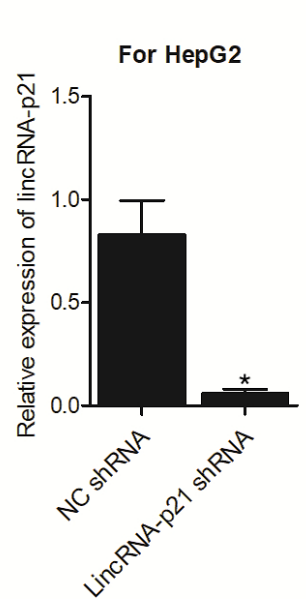

B

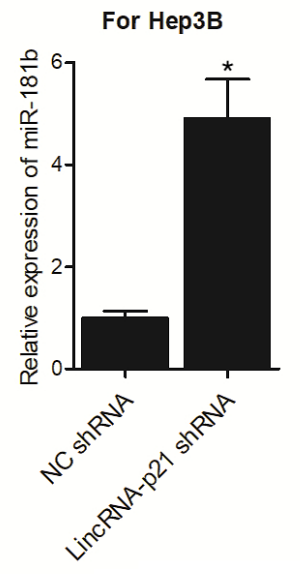

D
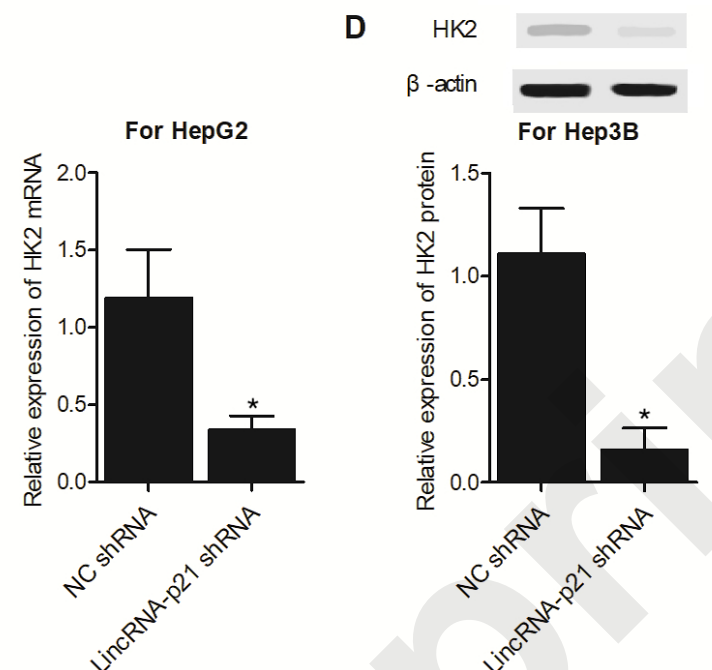

$\mathrm{F}$
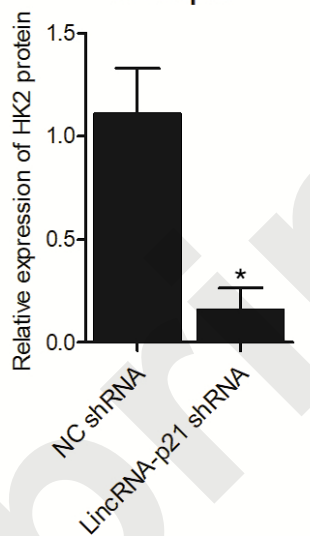

For Hep3B
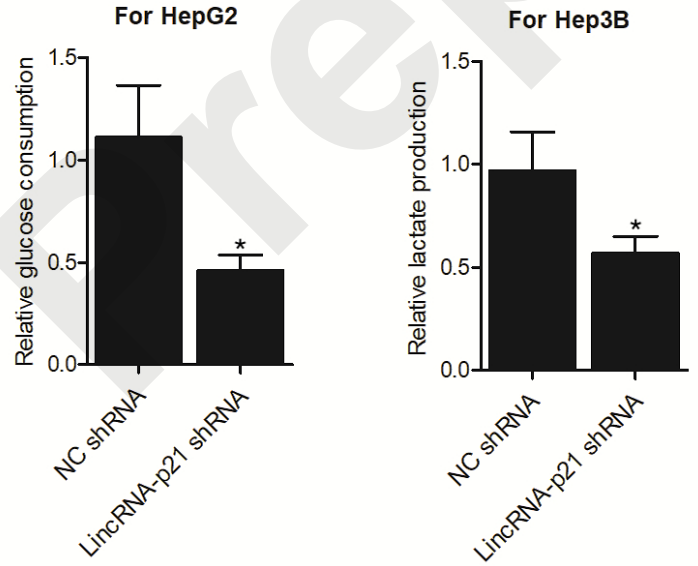
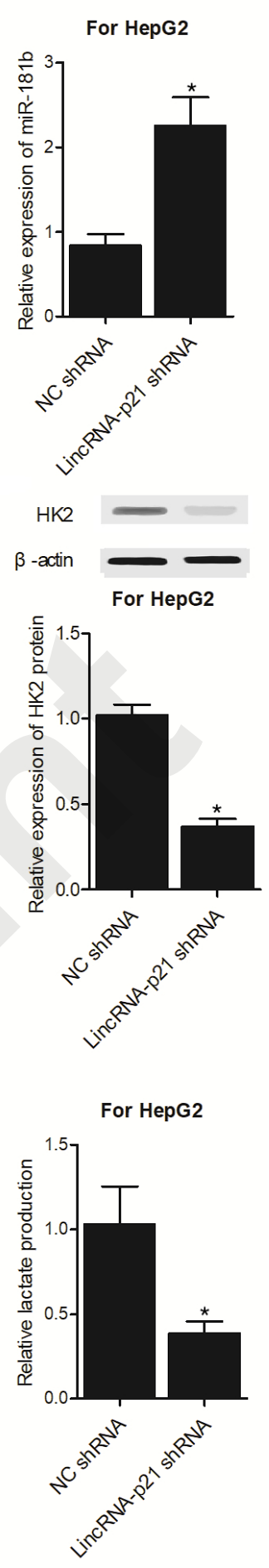

Fig.3

Suppression of lincRNA-p21 enhanced the expression of miR-181, repressed the expression of HK2 mRNA and protein, and reduced the levels of glucose consumption and lactate production ( ${ }^{*} \mathrm{P}$-value $<0.05$ compared with NC shRNA group; NC: negative control; NC shRNA functions as the control group).

A: Dramatic decrease of lincRNA-p21 expression in Hep3B and HepG2 cells transfected with lincRNA-p21 shRNA.

$B$ : The expression of miR-181b was remarkably increased by lincRNA-p21 shRNA in Hep3B and HepG2 cells. 
C: The expression of HK2 mRNA was remarkably decreased by lincRNA-p21 shRNA in Hep3B and HepG2 cells.

D: The expression of HK2 protein was remarkably decreased by lincRNA-p21 shRNA in Hep3B and HepG2 cells.

E: The glucose consumption was notably suppressed by lincRNA-p21 shRNA in Hep3B and HepG2 cells.

F: The lactate production was notably suppressed by lincRNA-p21 shRNA in Hep3B and HepG2 cells. 
A
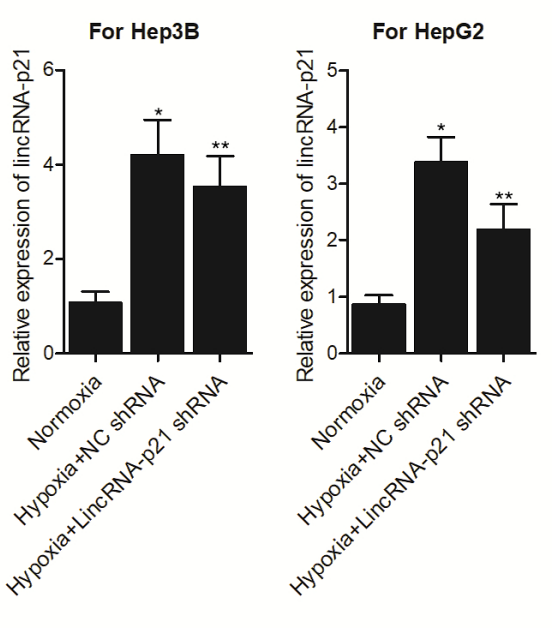

B

C
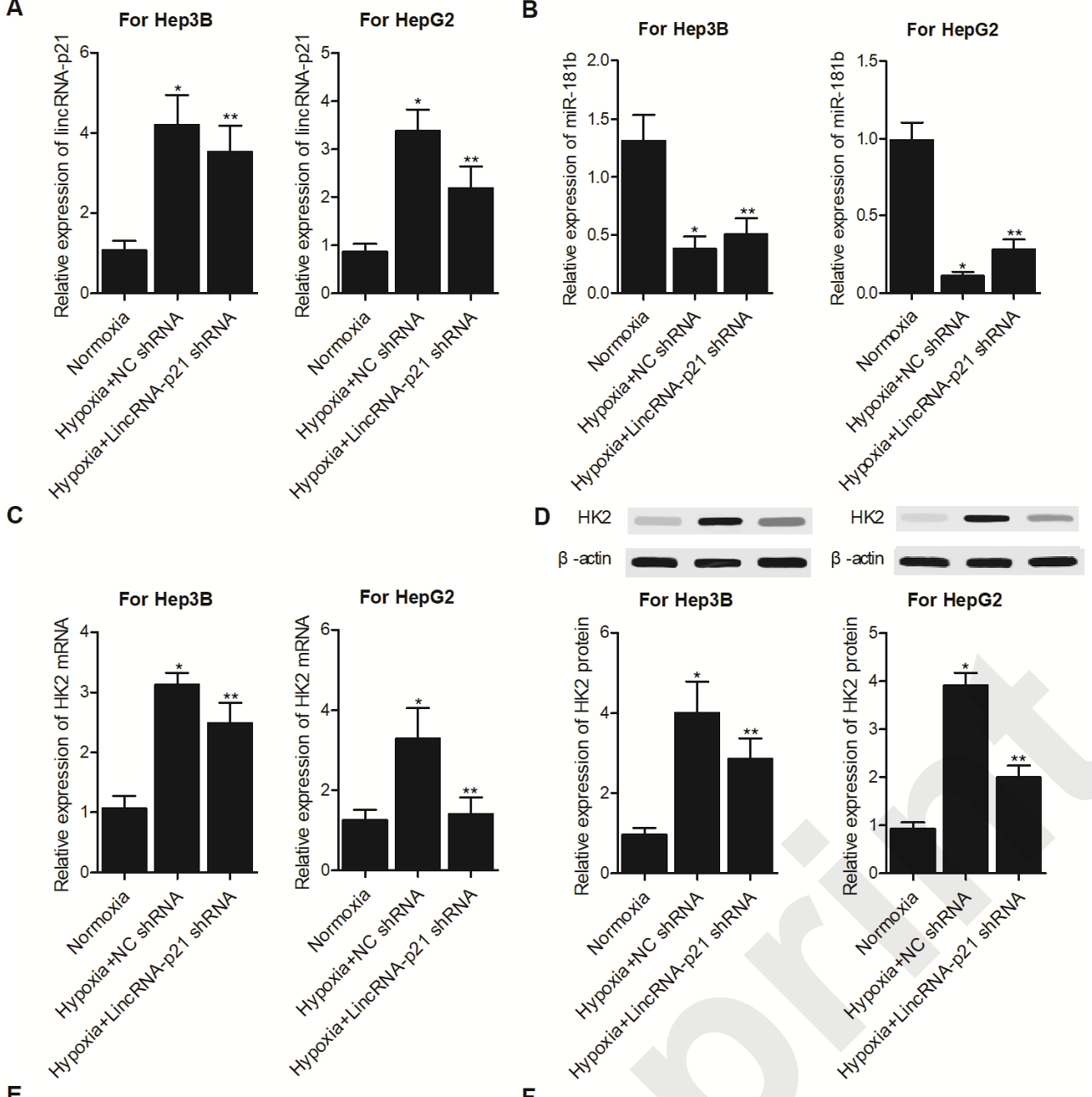

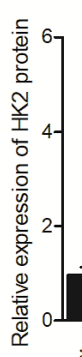

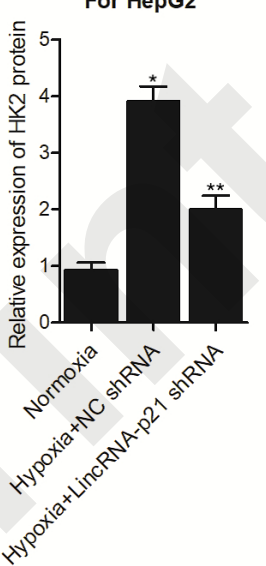

E
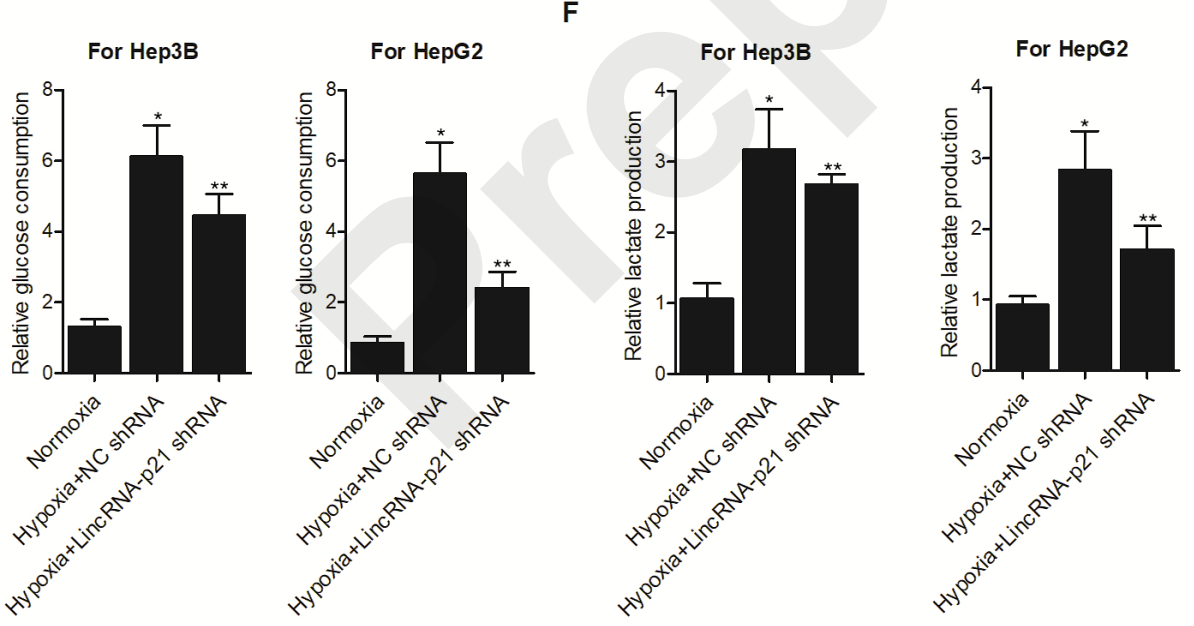

Fig.4

LincRNA-p21 shRNA attenuated hypoxia-induced dysregulation of miR-181b and HK2 expression, as well as the levels of glucose consumption and lactate production in Hep3B and HepG2 cells ( ${ }^{*} P$-value $<0.05$ compared with Normoxia group; ${ }^{* *} \mathrm{P}$ value $<0.05$ compared with Hypoxia + NC shRNA group; NC: negative control).

A: Hypoxia induced up-regulation of lincRNA-p21 was decreased by lincRNA-p21 shRNA in Hep3B and HepG2 cells.

B: Hypoxia induced down-regulation of miR-181b was decreased by lincRNA-p21 shRNA in Hep3B and HepG2 cells. 
C: Hypoxia induced up-regulation of HK2 mRNA was decreased by lincRNA-p21 shRNA in Hep3B and HepG2 cells.

D: Hypoxia induced up-regulation of HK2 protein was decreased by lincRNA-p21 shRNA in Hep3B and HepG2 cells.

$\mathrm{E}$ : Hypoxia induced elevation of glucose consumption was decreased by lincRNA-p21 shRNA in Hep3B and HepG2 cells.

$\mathrm{F}$ : Hypoxia induced elevation of lactate production was decreased by lincRNA-p21 shRNA in Hep3B and HepG2 cells. 


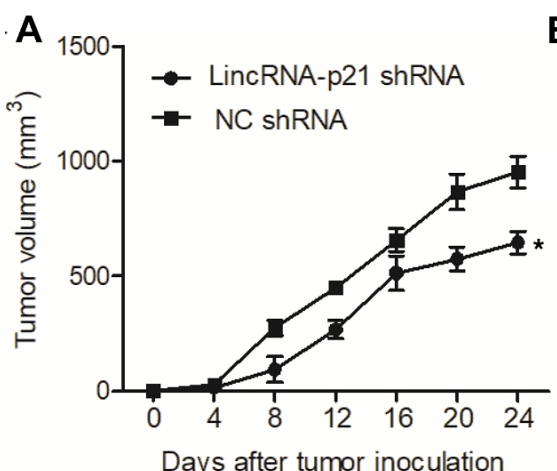

C

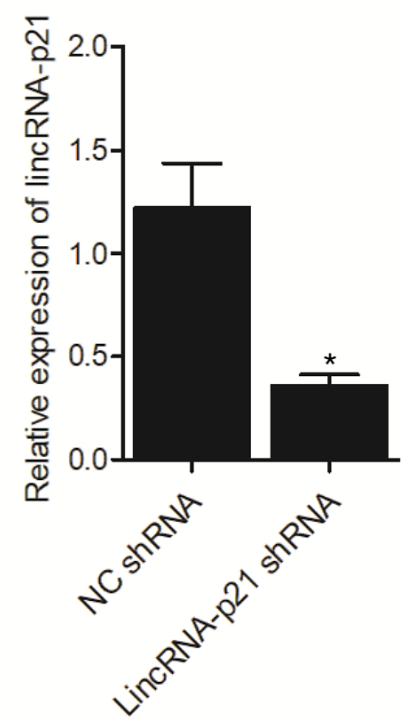

E

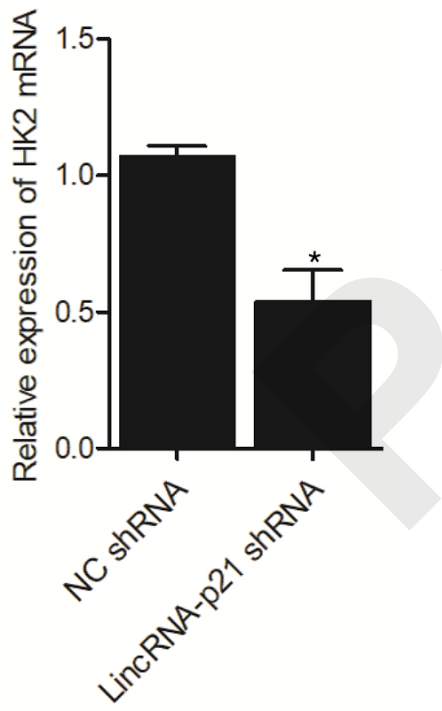

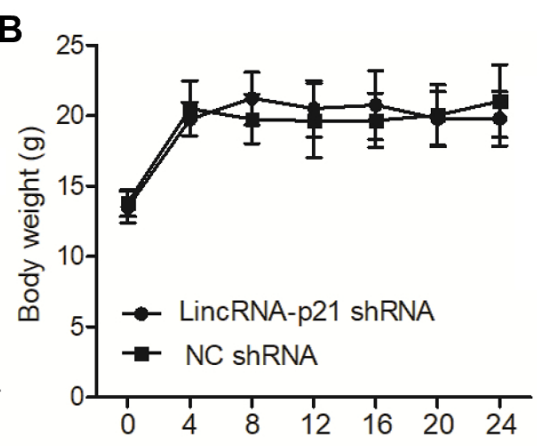

D

Days after tumor inoculation

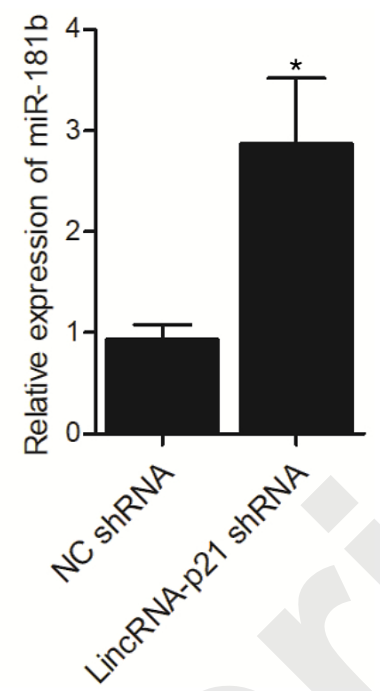

$\mathbf{F}$

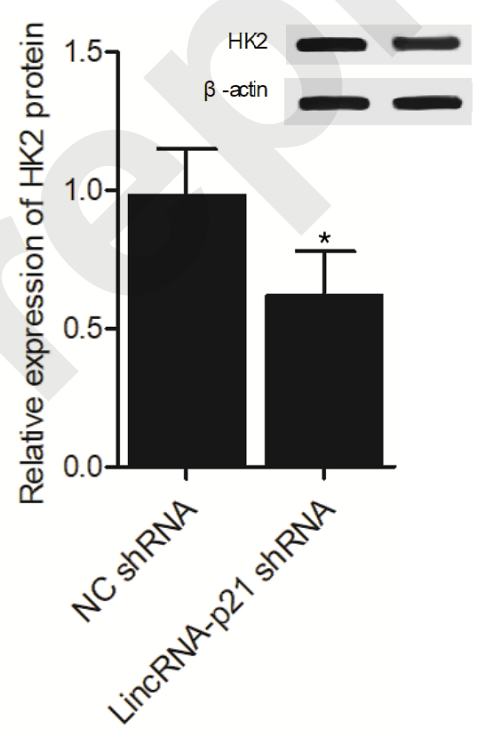

Fig.5

HepG2 cells treated by lincRNA-p21 shRNA altered the volume of tumor tissues and weight of nude mice, as well as the expression of miR-181b and HK2 ( ${ }^{*}$ P-value $<0.05$ compared with NC shRNA group; NC: negative control; NC shRNA functions as the control group). A: LincRNA-p21 shRNA remarkably decreased the tumor volume of the nude mice transplanted with HepG2 cells.

B: LincRNA-p21 shRNA did not significantly influence the body weight of the nude mice transplanted with HepG2 cells.

C: The expression of lincRNA-p21 was decreased in the HepG2 tumor tissue treated by 
lincRNA-p21 shRNA.

D: The expression of miR-181b was increased in the HepG2 tumor tissue treated by lincRNA-p21 shRNA.

$\mathrm{E}$ : The expression of HK2 mRNA was decreased in the HepG2 tumor tissue treated by lincRNA-p21 shRNA.

$\mathrm{F}$ : Western blot analysis showed that the expression of HK2 protein was decreased in the HepG2 tumor tissue treated by lincRNA-p21 shRNA. 

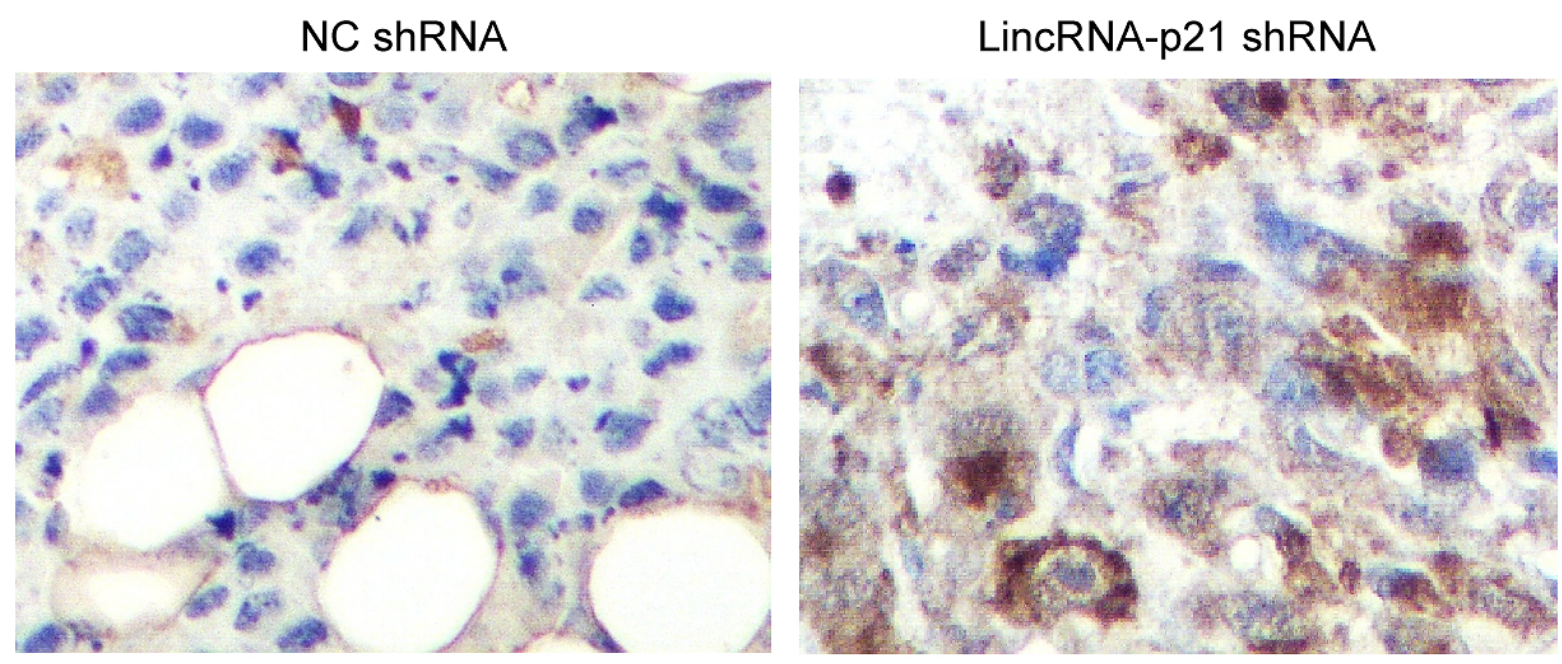

Fig.6

Immunohistochemistry analysis showed that the expression of HK2 protein was decreased in the HepG2 tumor tissue treated by lincRNA-p21 shRNA (The blue stain denotes the nucleus and the brown stain denotes the expression of target protein HK2). 